\title{
CRITERIOS PARA INTERNACIONALIZAR EL CURRÍCULUM
} UNIVERSITARIO

CRITERIA FOR THE INTERNATIONALIZATION OF UNIVERSITY CURRICULA

\author{
Volumen 12, Número 1 \\ Enero-Abril \\ pp. 1-25
}

Este número se publicó el 28 de febrero de 2012

Elsiana Guido Guido Ana Guzmán Aguilar

Revista indizada en REDALYC

Revista distribuida en las bases de datos:

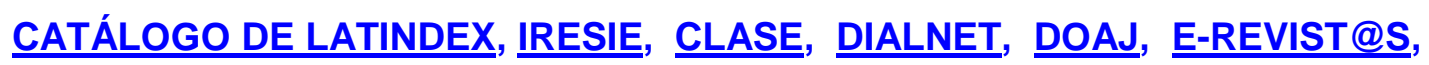

Revista registrada en los directorios:

ULRICH'S, REDIE, RINACE, OEI, MAESTROTECA, PREAL, HUASCARAN, CLASCO 


\title{
CRITERIOS PARA INTERNACIONALIZAR EL CURRÍCULUM UNIVERSITARIO

\author{
CRITERIA FOR THE INTERNATIONALIZATION OF UNIVERSITY CURRICULA
}

\author{
Elsiana Guido Guido \\ Ana Guzmán Aguilar
}

\begin{abstract}
Resumen: Este trabajo es producto de una investigación documental realizada en el año 2010 en el Centro de Evaluación Académica (CEA) de la Universidad de Costa Rica (UCR), acerca del tema de la internacionalización de la educación superior. Para esta investigación se entrevistó a personas del ámbito universitario relacionadas con el tema, se revisó bibliografía y se sistematizaron las experiencias en asesoría para el diseño curricular. Es a partir del análisis de esta información que se presenta una propuesta para alcanzar la internacionalización del currículo universitario considerando los intereses de la carrera, de la Universidad y con consistencia en todo el proceso formativo de la población estudiantil. Se visualiza la internacionalización como parte de la planeación universitaria, donde los actores del proceso asumen una posición crítica acerca de los beneficios y riesgos que conlleva. Además, la flexibilización curricular y el proceso formativo constituyen requisitos fundamentales para el desarrollo de la misma, ya que implica una apertura al cambio y la innovación, establecer contactos, comunicaciones y conexiones. La propuesta inicia con un proceso de reflexión a lo interno de la carrera, para determinar cómo y de qué manera aportarán al mejoramiento de la profesión y de los futuros profesionales; se privilegia, en los diferentes momentos del diseño curricular, las razones culturales y sociales que pueden impulsar a una carrera a tomar esta decisión, y se insiste en que la internacionalización debe ser parte de un proceso articulado en el transcurso de toda la formación.
\end{abstract}

Palabras clave: INTERNACIONALIZACIÓN, INTERNACIONALIZACIÓN DEL CURRÍCULUM, UNIVERSIDADES

Abstract: This article originated in a survey of documentation and bibliography carried out in 2010 at the Academic Evaluation Center of the Universidad de Costa Rica, around the topic of internationalization in higher education. Additionally, several persons in the university, who are related to this topic, were interviewed, and experiences of the authors were analyzed and considered. On this basis, this article proposes criteria for the internationalization of university curricula. It assumes that the internationalization process should be a part of regular university planning and should be informed by a critical perspective. It argues that the flexibilization of academic curricula should be an essential element for contemporary university planning, as it implies a favorable attitude towards change and innovation. The proposed criteria are framed within the ongoing internationalization of universities in the context of contemporary globalization. According to this proposal, the internationalization process should start with a reflection at each academic unit regarding how and in what way the profession and its future practitioners could benefit from internationalization. Finally, the article identifies social and cultural factors that may help foster a shift towards a more internationally-oriented curriculum.

Key words: INTERNATIONALIZATION, INTERNATIONALIZATION OF THE CURRÍCULUM, UNIVERSITIES

\footnotetext{
${ }^{1}$ Politóloga y Administradora de programas educativos no formales, Bachiller en Ciencias Políticas y Licenciada en Administración de programas educativos no formales, Universidad de Costa Rica. Investigadora del Departamento de Investigación y Evaluación Académica (DIEA) del Centro de Evaluación Académica (CEA) de la Universidad de Costa Rica. Dirección electrónica: elsiana.guido@ucr.ac.cr

${ }^{1}$ Licenciada en Enfermería y Magistra en Planificación Curricular. Profesora en la Escuela de Enfermería de la Universidad de Costa Rica y Asesora Curricular en el Departamento de Investigación y Evaluación Académica(DIEA) del Centro de Evaluación Académica (CEA) de la Universidad de Costa Rica. Dirección electrónica: ana.guzmanaguilar@ucr.ac.cr.cr
}

Artículo recibido: 16 de setiembre, 2011

Aprobado: 16 de febrero, 2012 


\section{Introducción}

El trabajo que se presenta es producto de la revisión documental del tema de la Internacionalización de la Educación Superior, realizada por las investigadoras del Departamento de Investigación y Evaluación Académica del Centro de Evaluación Académica (CEA) de la Universidad de Costa Rica (UCR), con el propósito de aportar algunos criterios en el diseño curricular de carreras que tengan interés en internacionalizar su currículum.

Se plantea una propuesta fundamentada en la concepción amplia del concepto internacionalización que abarca una mirada multidireccional, donde las carreras que se interesen por darle este enfoque a la formación de sus profesionales tienen la oportunidad de discutir y analizar las oportunidades y los riesgos que se visualizan con ella, por lo que deben definir su propia ruta.

De acuerdo con las investigadoras, los criterios de internacionalización del currículum, idealmente, deben partir del proceso de planificación universitaria y deben ser visualizados como parte integral del desarrollo institucional. Además, la reflexión y el análisis de los criterios de internacionalización en un diseño curricular implican el uso de un pensamiento crítico para determinar las consecuencias que tiene en el desarrollo de una unidad académica.

El propósito principal del estudio realizado en la UCR fue construir una propuesta que contribuyera a la incorporación en el currículum universitario del componente internacional, para lo cual se determinaron los objetivos específicos siguientes:

En relación con la internacionalización de la educación superior:

- Contextualizar el concepto de internacionalización de la educación superior, en sus dimensiones social, económica y política.

- Conocer las oportunidades y los desafíos que conlleva para las universidades, la internacionalización de la educación superior.

En relación con la internacionalización del currículum:

- Brindar algunas estrategias para promover la internacionalización del currículum en los planes de estudio de la Universidad de Costa Rica. 
La metodología utilizada fue la revisión bibliográfica de literatura actualizada, de documentos de la Universidad de Costa Rica, así como entrevistas con personas conocedoras del tema y la reflexión de las experiencias o lecciones aprendidas en asesoría curricular de las investigadoras.

Es así como, derivado de la investigación realizada, este artículo se estructura en dos partes: en la primera, se presenta el marco contextual de la internacionalización de la educación superior, donde se hace referencia a las condiciones económicas, políticas y sociales que favorecen e impulsan estos procesos y, en la segunda parte, se desarrolla la propuesta de internacionalización del currículum universitario.

\section{Marco contextual y conceptual de la internacionalización de la educación superior}

Se considera importante referirse al tema de la internacionalización de la educación superior como un proceso que permite comprender las acciones que se han desarrollado para propiciar la internacionalización del currículum en las universidades, por lo que, en este apartado, se resumen los contextos social, económico y político donde se desarrolla el proceso de internacionalización de la educación superior y se presentan algunas definiciones relacionadas.

Este concepto es comprendido por J. Knight (s.f.), citado en Quiang (2003), como: “el proceso de integrar la dimensión internacional/intercultural a las funciones de docencia, investigación y acción social de las universidades" (p.249). Esta definición implica un proceso dinámico y no tanto un conjunto de actividades aisladas que se encuentra estrechamente relacionado con las tres funciones sustantivas de las instituciones de educación superior.

Es importante destacar que se considera al proceso de internacionalización de la educación superior no como un fin en sí mismo, sino como una alternativa para responder a las demandas de la sociedad global; adicionalmente, se parte de una definición amplia del constructo que engloba, tal y como lo aclara Knight (s.f.), la dimensión intercultural del proceso, ya que la internacionalización no está solamente orientada a los estados-nación, sino que también incorpora a los diferentes grupos étnicos y culturales de un país. En este sentido, abogar por un proceso de internacionalización de las universidades, basado 
únicamente en la geografía, es una visión limitada, ya que una institución puede tener múltiples causas o motivos para internacionalizarse.

La internacionalización de la educación superior, desde el punto de vista de Madera (2005), se convierte en una estrategia de carácter transversal que permite la transferencia de saberes y recursos que facilitan este cambio.

Por su parte, Beneitone, en su exposición en el Foro sobre Internacionalización de la Educación Superior, realizado en la Universidad del Cauca, en marzo de 2008, dice que

La Universidad observa críticamente la globalización, se apropia de sus aspectos positivos, recodificando los valores y tendencias que apartan el sistema mundial del desarrollo humano efectivo para todos. Trasciende el mercantilismo educativo y social, la competitividad dura, las relaciones internacionales, a favor de la construcción de la Sociedad del Conocimiento apoyada por una cooperación solidaria, horizontal, propiciando pertinencia, calidad equidad y accesibilidad.

De este modo, la relación entre globalización, entendida concretamente para la educación superior, y la internacionalización la describe Knigt (s.f., đ 5) de la siguiente manera

La globalización es el flujo de tecnología, economía, conocimientos, personas, valores, ideas... a través de las fronteras. Afecta a cada país de manera diferente en virtud de la historia, las tradiciones, la cultura y las prioridades de cada nación. La internacionalización de la educación superior es una de las maneras en que un país responde a las repercusiones de la globalización, no obstante que respeta la idiosincrasia de la nación. Por ende, ambos conceptos, aunque distintos, están vinculados dinámicamente. La globalización puede considerarse como el catalizador, en tanto que la internacionalización es la respuesta, si bien, una respuesta proactiva.

A pesar de la estrecha relación manifiesta entre los procesos de globalización y la internacionalización de las universidades, es importante diferenciar ambos conceptos, ya que no hacen referencia a los mismos procesos en las universidades.

Tünnermann (s.f.) propone la siguiente distinción entre internacionalización de la educación superior y transnacionalización del sector educativo

Mientras en la internacionalización se propugna, siguiendo los lineamientos de la Declaración Mundial sobre la Educación Superior, por una cooperación internacional 
solidaria con énfasis en la cooperación horizontal, basada en el diálogo intercultural y respetuosa de la idiosincrasia e identidad de los países participantes, así como el diseño de redes interuniversitarias y de espacios académicos ampliados, en la transnacionalización se trata de facilitar el establecimiento en nuestros países de filiales de Universidades extranjeras, de una cooperación dominada aun por criterios asistenciales, así como la venta de franquicias académicas, la creación de universidades corporativas, auspiciadas por las grandes empresas transnacionales, los programas multimedios y las universidades virtuales, controladas por universidades y empresas de los países más desarrollados. Este nuevo panorama que comienza a configurarse en nuestros países, ha hecho surgir voces de alerta por el riesgo que representan para nuestra soberanía e identidad nacional. (p. 4)

Tunnermann (s.f.) menciona, además, que la internacionalización tiene muchos más beneficios para las educación superior que la transnacionalización, ya que

... se caracteriza por inspirarse en principios de solidaridad, como lo establece la Declaración Mundial, reúne a la cooperación horizontal tanto como a la Norte-Sur, privilegia los hermanamientos entre Universidades, las redes académicas y la constitución de espacios académicos ampliados. En cambio, la transnacionalización prioriza el establecimiento de filiales en el extranjero de las Universidades de los países más avanzados y el sistema de franquicias. (p. 6)

Estas reflexiones de Tunnermann hacen, entonces, un llamado a la comprensión y al análisis de los conceptos, tomando en consideración las perspectivas social, política y económica de las Universidades y del país.

\section{Algunos motivos para la internacionalización de las universidades}

En la literatura se encuentran diferentes motivaciones por las cuales se desea impulsar la internacionalización de la educación superior. Estas diferentes motivaciones, o incentivos, se han presentado a lo largo de la historia dependiendo del momento histórico y del contexto económico imperante. Es así como Knight, citada por Qiang (2003, p. 252) clasifica en cuatro las razones por las que las universidades desean internacionalizarse. 
En primer lugar se encuentran las razones políticas, que hacen referencia a la posición del país y a su rol en el mundo, donde la educación superior desempeña un papel importante en el posicionamiento mundial del país. Aquí, cobran relevancia estratégica los conceptos de seguridad, paz e influencia ideológica, entre otros. De este modo, la internacionalización contribuye al posicionamiento político del país y las becas a estudiantes son consideradas, en este sentido, como una "inversión política", ya que las personas becadas, consideradas líderes, regresan a sus países de origen con "un mayor conocimiento y simpatía hacia el sistema político, la cultura y los valores del país que les acogió durante su formación".

También, se hace referencia a las razones económicas que tiene un país donde la internacionalización de la educación superior se visualiza como una manera de promover la competitividad internacional de la nación y contribuir al desarrollo económico y tecnológico. Desde una perspectiva institucional, una universidad puede recibir importantes recursos financieros derivados del pago de los estudiantes extranjeros matriculados, lo cual es una orientación muy importante en el caso de algunos países europeos y de algunas universidades en Norteamérica. Esta es la tesis de autores como Rafael Pedregal (2003), quien analiza las implicaciones económicas en el Tratado de Libre Comercio entre México, Canadá y los Estados Unidos y alerta sobre las causas económicas como el principal impulsor de la internacionalización de la educación superior en México.

Así lo explica Knight, (s.f., I 24) cuando afirma que: “algunos métodos eficaces para mejorar y mantener la competitividad son: el desarrollo de un personal bien capacitado y bien informado, y la inversión en la investigación aplicada". Ambas estrategias implican al sector de educación superior y, por ello, en los ámbitos nacional y regional, la internacionalización del sector de la educación superior y el desarrollo económico y tecnológico estrechan sus vínculos cada vez más.

En tercer lugar, se pueden mencionar las razones académicas que se refieren a la consecución de estándares académicos internacionales para la docencia, la investigación y la acción social, que además son importantes en los procesos de acreditación y reconocimiento nacional e internacional. Gacel-Ávila (s.f.) agrega que el intercambio internacional se visualiza como un valor agregado a la calidad de la educación superior, ya que permite a los/las docentes y a estudiantes continuar su formación académica en otras universidades que ofrecen opciones diferentes; también, favorece la actualización de docentes y de planes de estudio si el intercambio se realiza con instituciones cuya calidad 
sea reconocida (p. 32). Knight (s.f., \ 28) aclara que esta razón académica está relacionada directamente con la historia y el desarrollo de las universidades, a la vez, hace un llamado a mantener una actitud crítica con este enfoque al afirmar que

La movilidad de profesionales y la dimensión internacional en la investigación existen desde hace cientos de años. $Y$ al mismo tiempo, conviene tomar en cuenta las nuevas tendencias, una de las cuales es la influencia del enfoque de mercado en la educación superior y el énfasis en la calidad tanto desde la perspectiva de mejoramiento como de la responsabilidad. Una de las principales razones aducidas para internacionalizar al sector educativo es lograr estándares académicos internacionales en la enseñanza y la investigación, tema de intensas controversias. Preocupan la uniformidad y homogeneidad que resulten a causa del énfasis excesivo en los estándares internacionalmente aceptados. Son muchos y complejos los factores que entran en juego en este debate, y cabe tomar en cuenta las preocupaciones acerca de utilizar un enfoque de "todos cortados con el mismo molde" con respecto a la educación, aunque no por ello se dejen de alcanzar estándares de excelencia internacional en las becas y la investigación.

Finalmente, se tienen las razones culturales y sociales que propician el proceso de internacionalización de la educación superior. Este aspecto se refiere al papel y el lugar que tiene la propia cultura y el idioma para el país, así como a la importancia de entender idiomas y culturas extranjeras (Knight, s.f.). Dentro de este último punto Qiang (2003) considera que la preservación y promoción de la cultura nacional es una motivación fuerte para aquellos países que consideran a la internacionalización como un camino para respetar la diversidad cultural y servir de contrapeso al efecto de homogenización que se percibe con la globalización. (p. 253) (traducción libre).

John Mallea, citado por Pedregal (2003), señala que el principal motivo para que la educación superior se internacionalice se debe a:

los retos planteados por la aparición de una economía cada vez más competitiva y global" (p. 31), además de las siguiente razones una forma de diplomacia cultural, una forma de reducir la percepción política y cultural de aislamiento, un vehículo para promover el entendimiento internacional entre sus ciudadanos, una obligación moral de 
los países desarrollados de proveer ayuda a los países en desarrollo más allá de sus fronteras (p. 31).

De esta manera, tal y como lo resume Gacel-Ávila, (s.f.)

El fin último de la internacionalización es promover la convivencia social y cultural, la paz entre todos los pueblos del mundo; también lo es la erradicación de la miseria y de la hambruna, así como la contención del deterioro creciente del medio ambiente y los problemas de salud pública, entre otros (p.33).

Se tiene, entonces, que la internacionalización se plantea como una oportunidad para que las universidades se replanteen este tema, tomen posición acerca de las principales motivaciones que tienen para internacionalizar su oferta curricular y valoren tanto los beneficios como los riesgos que implican estos procesos, tanto para las universidades como para las carreras propiamente.

Para la Universidad de Costa Rica y la Oficina Asuntos Internacionales (OAI) (2011), la internacionalización de su oferta académica puede significar un importante crecimiento académico, el fortalecimiento de las redes académicas internacionales, la promoción de la movilidad en todas sus formas mediante becas, pasantías y aprendizaje de otros idiomas.

Asimismo, y como lo afirma Ana Sittenfeld, (2010), desde esta Oficina se han buscado estrategias para avanzar en este aspecto e incidir más en la gestión del currículo universitario y no ser solo un ente que reparte becas al extranjero. Sin embargo, la posición que se mantiene en este artículo es que estos esfuerzos son importantes, pero no suficientes, ya que es necesario ampliar el ámbito de posibilidades para articular, tal como lo proponemos, otros elementos que impacten específicamente los procesos pedagógicos y de gestión del currículum para lograr que la internacionalización sea parte de la formación de los futuros profesionales de una carrera y no solo la experiencia de algunas personas.

En un estudio realizado en el 2005 por Knight para la Global University Network for Innovation, GUNI, (citado por la Japan Society for the Promotion of Science), para el que encuestó a instituciones de educación superior de 95 países, se concluye que para la mayoría de las directivas y los directivos de las universidades, la internacionalización de la educación superior tiene grandes potencialidades y beneficios, así como riesgos. 
Dentro de los beneficios se mencionaron a estudiantes y funcionarios más orientados hacia una perspectiva internacional, el mejoramiento de la calidad académica, el fortalecimiento de la investigación y la producción de conocimiento, innovación en el currículum, en la docencia y en la investigación, promoción de mayor entendimiento y solidaridad internacional. Dentro de los riesgos se señalaron los siguientes: homogenización y comercialización de los programas educativos, incremento en el número de títulos extranjeros y/o proveedores de baja calidad, pérdida de cerebros, crecimiento elitista en cuanto al acceso a las oportunidades de educación internacional, sobreuso del inglés como un medio de instrucción, y pérdida de la identidad cultural y nacional (p. 82).

Tal y como lo resume Fedorov (2009), la internacionalización de la educación superior no debe tratarse como una panacea, a pesar de sus reconocidos beneficios. Dentro de los riesgos y desafíos que se señalan, el más notorio es el que se deriva de una cierta posición predominante, dentro de la tendencia mercantilista del proceso, donde la internacionalización se asume como un camino o alternativa de intervención ideada por las instituciones de los países desarrollados, hacia los países con menos desarrollo. Es en este sentido que se hace un llamado a que el proceso de internacionalización se de en varias vías e intencionalidades, y que no se apoye únicamente en la influencia de los países del norte.

De esta manera, las personas encargadas en las universidades y, concretamente, las carreras universitarias, deben analizar los beneficios y los riesgos asociados con los procesos de internacionalización. Además, deben asegurarse de que todas la acciones realizadas con este objetivo estén encaminadas a articular los esfuerzos en la gestión universitaria.

\section{La internacionalización de la educación superior como parte de la planeación universitaria}

Tal y como lo afirma Gacel-Ávila, (s.f.), la internacionalización de la educación superior no se visualiza hoy en día como actos individuales de intercambios de estudiantes y de docentes, o de movilidad de personas sin mayor repercusión para la institución educativa. Actualmente, este tema se presenta como parte del interés institucional, ya que en

las nuevas políticas educativas, la colaboración internacional se convierte en un poderoso y estratégico instrumento para elevar la calidad de la educación, para preparar a los egresados a funcionar en un mundo cada día más interdependiente y 
competitivo, y para crear mayor comprensión y solidaridad entre los pueblos del planeta. La internacionalización es una necesidad insoslayable y un fenómeno irreversible. Al internacionalizarse, la universidad accede a su vocación primera, la de la universalidad. (p. 29)

De acuerdo con la autora, con la internacionalización de la educación superior se deben retomar dos aspectos fundamentales: por un lado, recomienda que sea vista como un proceso de transformación institucional, donde se van integrando la dimensión internacional a la misión y funciones sustantivas de la universidad. Esto implica, por un lado, el desarrollo de estrategias administrativas y académicas para operacionalizar el proceso y, por otro lado, la internacionalización de la docencia, la investigación y la acción social. En este recorrido se sugiere que estudiantes, personal académico y administrativo tengan las habilidades, conocimientos y actitudes que les permitan desempeñarse en un medio internacional y multicultural (p. 31).

Gacel-Ávila (s.f.) plantea, de manera muy clara, este nexo entre los procesos de internacionalización y la planeación universitaria, al escribir que esta

se refiere a un proceso de transformación institucional integral que pretende incorporar la dimensión internacional e intercultural en la misión y las funciones sustantivas de las instituciones de educación superior, de tal manera que sean inseparables de su identidad y cultura. El proceso de internacionalización debe ser visto como una apertura institucional hacia el exterior y debe ser parte integral de los planes de desarrollo, planeación estratégica y políticas generales de las instituciones. Pero para lograr este objetivo es preciso que exista dentro de las instituciones de educación superior una política internacional explícita, claramente definida y respaldada por las autoridades universitarias, consensuada en la comunidad universitaria e implementada por una estructura administrativa y académica apropiada para su organización y promoción adecuada. (p. 30)

En resumen, la internacionalización de la educación superior se visualiza como una respuesta a los procesos de globalización de las distintas esferas de las sociedades. Este proceso se convierte en una estrategia de carácter transversal que permite la transferencia de saberes y recursos que facilitan posicionarse ante la tendencia globalizante, ya que las reflexiones que las carreras realicen en torno a este tema puede favorecer el surgimiento de 
consensos alrededor de esta temática y generar una nueva mirada al impacto de la globalización, por lo que la internacionalización se plantea como un eje que articula en lugar de ser una experiencia aislada (Madera, 2005).

\section{Internacionalización del currículum universitario}

De acuerdo con Inmaculada Madera (2005), un currículum internacionalizado se refiere a la integración de la dimensión internacional en el proceso docente educativo: contenidos y formas de los programas de curso, métodos didácticos, sistemas evaluativos, investigación y extensión, criterios de calidad, concepto de pertinencia, cobertura y equidad. A través de la incorporación de esta dimensión en la cultura de las organizaciones educativas, se pretende formar sujetos capaces de actuar social y profesionalmente en un contexto internacional, interdisciplinario y multicultural. (p. 3)

Desde esta perspectiva, el currículum internacionalizado se concibe como la incorporación de la dimensión internacional en todos los componentes de la propuesta curricular y no únicamente en el planeamiento de los cursos, además, atiende no solo a una oportunidad en el ámbito económico para los profesionales, sino también a una oportunidad de crecimiento sociocultural de los individuos y las instituciones, un aspecto que coincide con el enfoque de la propuesta que se expone, más adelante, en este artículo y los intereses de la Universidad de Costa Rica.

Otra autora que concuerda con esta manera de comprender la internacionalización del currículum es Alicia Camillioni (2010), quien sostiene que se trata de una fuerte tendencia que implica incorporar experiencias interculturales, de diferentes maneras, para aprender a convivir con otros.

Es así como en esta propuesta se visualiza la internacionalización del currículum no como una materia especifica dentro del plan de estudios, sino como la conjunción de contenidos y métodos de enseñanza que le permitan al estudiantado explorar la perspectiva internacional dentro de su disciplina, comprender y respetar la diversidad nacional y la de otros países, desarrollando competencias comunicativas que le permitan responder de manera creativa y respetuosa a nuevas formas de interrelación.

Dentro de este contexto, la oferta educativa de una carrera debe procurar el fomento de competencias interculturales e internacionales tanto en su propia universidad como en el 
extranjero garantizando, mediante la gestión del currículum, la comprensión de la interdependencia de las diversas culturas y ambientes.

\section{Criterios para internacionalizar el currículum}

Los criterios para lograr la internacionalización han sido estudiados por diversos autores, tales como Madera (2005), Gacel-Ávila (2007) y Bebeitone, Esquetini, González, Maletá, Siufi, y Wagenaar. (2007) quienes coinciden en que estos se deben incorporar según las características de los contextos educativos, posibilidades y oportunidades concretas, políticas universitarias y de las situaciones sociales y políticas vigentes.

En materia de currículum es importante reiterar que la internacionalización debe ser parte del proyecto educativo y, como tal, reflejarse en la propuesta curricular de la unidad académica. De acuerdo con lo que plantea Ruiz (2005), el proyecto educativo de la carrera define la identidad de la institución, es un proceso progresivo y dinámico que permite la consolidación de los equipos docentes y facilita la gestión del currículo, atiende a las formas de organización y coordinación de los procesos, al organigrama, a las reglas de funcionamiento, a los valores y al modelo de persona y sociedad que propician, por tanto, debe gestarse, también, una intención para valorar la importancia de ampliar horizontes y acoger nuevas experiencias que los impulse a compartir su saber con el resto del mundo y a la vez ser parte de otros saberes y estar con otras culturas para incorporarlo a su práctica cotidiana.

La internacionalización debe pasar primero por la inserción en el proyecto educativo para que, desde los diferentes procesos organizativos, se puedan articular estrategias en el plan de estudios; las propuestas de internacionalización se deben adaptar a contextos específicos, y es competencia de los docentes garantizar la coherencia y la coordinación de estas iniciativas para que favorezcan el desarrollo de los y las estudiantes.

\section{Consideraciones para la internacionalización del currriculum}

\subsection{Primero la flexibilización}

La flexibilización del currículum es el primer paso para desarrollar estos criterios de internacionalización. Esta corriente procede, a su vez, del movimiento de la globalización, que ha incidido, según María Eugenia Upegui (2007), en el sistema educativo impulsándolo a la apertura y al incremento de relaciones con la sociedad. Es importante comprender este 
contexto para que se facilite la manera en que se pueden implementar estos criterios en los procesos educativos (Orozco, 2009).

La flexibilidad curricular, según lo plantea esta autora, implica una apertura al cambio y la innovación, estar abiertos a lo diverso y a tratar con el otro o lo otro; esta apertura significa remover fronteras, barreras, restricciones y establecer contactos, comunicaciones y conexiones. El tema se relaciona, también, con la capacidad de enfrentarse a situaciones cambiantes del conocimiento y la sociedad.

Sin embargo, la noción de currículo flexible tiene múltiples significados y se trata de diversas formas en las organizaciones académicas, que como bien lo explica Orozco (2009), son un espacio de tensión entre los diversos intereses políticos que desean intervenir, provocan también nuevos sentidos y re-significados del discurso y propician nuevas creaciones que parten de la propia cultura y política socioeducativa. Es responsabilidad de quienes trabajan en currículum analizar la variedad de significados que se dan a estos conceptos y adaptarlos en las intervenciones que se hacen en la construcción, diseño o restructuración de un plan de estudios, analizando las implicaciones y las posibilidades académicas de estas propuestas.

Según Canudas (2006) la flexibilidad del currículo es una alternativa a la rigidez e inmovilidad de la formación universitaria que anula la posibilidad de desarrollar capacidades individuales, esto se entiende desde varias perspectivas tales como:

- Flexibilidad en el tiempo de formación: incorporando cursos nuevos o actividades específicas sin alargar demasiado el periodo de graduación.

- Flexibilidad en la especialización: las especialidades son conjuntos de cursos destinados a capacitar al estudiante para que se desenvuelva mejor en una determinada área de su profesión.

- Flexibilidad para la adaptación a nuevos currículos, tomando del nuevo currículum sólo los créditos necesarios para completar la cuota establecida.

Estas son posibles alternativas para propiciar la flexibilidad, ya que este principio se basa en que la educación debe enfocarse en el aprendizaje de formas y métodos de pensamiento que favorezcan la formación integral y autónoma del estudiantado, donde se consideren participantes activos y activas; por lo tanto, debe romperse con la visión lineal y 
rígida de los planes de estudio tradicionales y propiciar una mayor gama de opciones para la formación profesional (Perdomo, s.f.).

De este modo, la flexibilidad es poder anticiparse a los problemas que surgen del gran dinamismo del mundo académico en el dominio de la disciplina y de la profesión, por lo que se debe ser versátil para incorporar esos cambios.

\subsection{Otros Criterios}

En la literatura se sugieren tanto criterios generales para internacionalizar el currículum, como estrategias especificas para operativizar este enfoque en el plan de estudios. Para el contexto de la Universidad de Costa Rica, se proponen los siguientes criterios como guía para las carreras que deseen internacionalizar su propuesta curricular, (basado en algunas de las ideas expuestas por Betty Leask, 1999 y Xiomara Zarur, 2010).

Un currículum internacionalizado le posibilita al estudiante:

1. Abordar las temáticas de manera global y considerar diversas perspectivas culturales en el análisis de los problemas.

2. Tener conciencia de su propia cultura y de otras culturas.

3. Reconocer los temas interculturales relevantes para su ejercicio profesional.

4. Valorar la diversidad de idiomas y culturas.

5. Aplicar estándares y prácticas de la disciplina, aceptadas internacionalmente.

6. Matricular cursos en su país, ofrecidos de manera conjunta con otra (s) universidad (es) extranjera (s).

7. Participar en programas interdisciplinarios, donde participen universidades extranjeras.

8. Prepararse en su propia universidad mediante experiencias educativas ofrecidas en otros idiomas, donde participen estudiantes de diversos países.

9. Interactuar con docentes de su universidad que aplican estrategias didácticas que ayudan al estudiantado a ampliar su perspectiva intercultural de la profesión y la disciplina.

10. Interactuar con docentes de su universidad que mantengan contactos profesionales internacionales y se mantengan actualizados acerca de las prácticas de calidad internacionalmente aceptadas en la profesión. 
11. Participar en investigaciones y proyectos socioculturales con estudiantes y docentes de universidades extranjeras.

12. Formarse en una carrera que tenga los requisitos de calidad internacionalmente aceptados para la profesión.

13. Compartir con estudiantes extranjeros matriculados en su universidad.

14. Tener la posibilidad de realizar prácticas, pasantías o cursos en el extranjero.

15. Compartir con profesores /as extranjeros invitados por su universidad.

16. Matricular cursos de su carrera que se ofrezcan en otros idiomas.

17. Contar con servicios de apoyo y gestión que posibiliten sus experiencias interculturales.

18. Acceso a información y aprendizaje virtual mediante tecnologías informáticas que fomenten el intercambio cultural.

\section{Propuesta de criterios para internacionalizar la propuesta curricular de las Unidades Académicas en la Universidad de Costa Rica}

Para elaborar la propuesta de los criterios que se pueden tomar en cuenta para internacionalizar el currículum, se ha tomado como referencia los momentos del diseño curricular elaborados por Carolina Bolaños, Alicia Vargas y Leda Velázquez (1999) titulado Hacia un diseño curricular alternativo en la educación superior, en el que las investigadoras proponen los criterios que deben considerarse en un diseño a partir de una definición conceptual de currículum y de una posición analítica y reflexiva para abordar estas situaciones. Bolaños et al. (1999) toman de la curriculista mexicana Alicia de Alba la siguiente noción de currículum "como la síntesis de elementos culturales, que se concretan en un proyecto político educativo, construido por la interacción de grupos con diversos intereses y visiones de mundo, que responden a su propia dinámica sociohistórica" (p. 185).

Las autoras citadas plantean un diseño curricular que contempla seis grandes fases, a saber: el marco socioprofesional de la disciplina, el marco epistemológico, el enfoque pedagógico, la organización o estructura de los saberes y la administración ejecución y seguimiento.

La presente propuesta de criterios para internacionalizar el currículum se basa en una serie de preguntas generadoras que pretenden impulsar el razonamiento o pensamiento crítico, un aspecto clave que coadyuva al éxito de estos procesos. Autoras como Alfaro 
(1996), exponen que: "el pensamiento crítico es la clave para resolver problemas" (p. 4), ya que se trata de una habilidad que permite adaptarnos a nuevas situaciones, tomar decisiones competentes y aprender constantemente. Otras definiciones del término nos dicen que

El pensamiento crítico es un pensamiento que tiene propósito (probar un punto, interpretar lo que algo significa, resolver un problema)... es una tarea colaborativa no competitiva, [las personas] trabajan en un proceso conjunto y pueden ayudarse mutuamente para llegar a la verdad. (Facione, 2007, p. 3)

De ahí que en la propuesta de Bolaños, Vargas y Velásquez (1999), al partir de preguntas generadoras se impulsa una forma de abordar el diseño curricular que sugiere poner en práctica habilidades cognitivas como la interpretación, el análisis, la evaluación, la inferencia, la explicación y la autorregulación, un elemento importante que implica cuestionar, confirmar o corregir el razonamiento propio. Todo esto para pensar efectivamente sobre algo que es en primera instancia la reflexión sociohistórica y epistemológica de la disciplina, presentando una serie de preguntas que ayudan al cuerpo docente de las unidades académicas a posicionarse sobre el tema, que no quiere decir ponerse de acuerdo con un único pensamiento, sino lograr consenso para desarrollar la propuesta y, de igual manera, abordar los otros elementos del diseño relacionados con la gestión del currículo.

Es así como sugerimos, a manera de complemento y partiendo de este principio de criticidad frente a los discursos ideológicos y de grupos sociales involucrados en los cambios curriculares, algunas otras preguntas que pueden guiar la discusión en las carreras que deseen internacionalizar su currículum; estas interrogantes no pretenden ser exhaustivas, pero sí intervenir en todos los componentes de la reflexión curricular para lograr la coherencia y consistencia en la propuesta, superando la visión de internacionalización del currículum como solamente el planeamiento de algunos cursos específicos o el intercambio de estudiantes.

\subsection{En el proyecto educativo de la unidad académica}

Como se mencionó en el apartado anterior, el proyecto educativo de una institución se refiere a la gestión y a la organización administrativa que permite darle un rumbo a esta, por 
lo que el liderazgo y la voluntad política son fundamentales para dar paso a estas propuestas diferentes.

Para incursionar en el campo de la internacionalización en el ámbito de la Universidad de Costa Rica, es prioritaria la determinación del cuerpo docente y administrativo de la unidad académica, de asumir una posición de apertura a esta iniciativa, pues hasta la fecha no es un mandato institucional.

Para realizar este tipo de gestiones, la unidad académica debe reflexionar en torno a las siguientes cuestiones:

1. ¿Se pueden establecer mecanismos para el reconocimiento y equiparación de cursos que son aprobados en el extranjero?

2. ¿Es factible implementar procesos de aprendizaje de una segunda lengua para docentes y estudiantes?

3. ¿Qué recursos son necesarios para la divulgación del quehacer académico y científico en revistas internacionales que hagan visible la unidad académica en ámbitos internacionales?

4. ¿De qué manera se pueden desarrollar procesos de actualización permanente en idiomas?

5. ¿En el plan de estudios se pueden promover actividades que permitan el acercamiento de diversas culturas al quehacer de la profesión, tal como conferencistas invitados para una temática especifica, cursos en inglés u otro lengua, impartidos de manera que estudiantes y docentes lo compartan y lo ejerciten?

6. ¿Se puede incorporar en los cursos literatura en otros idiomas, así como referencias bibliográficas?

7. ¿Qué mecanismo de articulación se debe implementar para establecer un nexo entre los y las docentes que tengan salidas al exterior con las temáticas de los distintos cursos de la carrera?

8. ¿Cómo se beneficiará la población estudiantil de las experiencias de internacionalización?

9. ¿Cómo estimular, en la investigación, la participación de las poblaciones docentes y estudiantiles que realicen pasantías o posgrados, y que puedan dar un aporte desde su aprendizaje internacional a estos procesos del grado? ¿Cómo incluirlos como personas que realizan lectorías, tutoras o directoras de tesis? 
Es aquí donde la flexibilidad es un aspecto fundamental que implica determinar el nivel, grado o criterio con que se van enfrentar los intercambios de docentes, estudiantes, saberes, culturas, conocimientos, etc. La apertura y el incremento de relaciones con otros contextos implican una actitud receptiva al cambio y la innovación.

\subsection{En el diseño curricular}

\subsubsection{Marco socioprofesional}

Bolaños et al., (1999) definen este marco como: "la explicitación de la evolución sociohistórica que ha tenido la disciplina o la profesión en los últimos años, tanto en el ámbito nacional, internacional e institucional. Incluye la contextualización, justificación y la caracterización profesional' (p. 188).

Este apartado propicia una revisión en retrospectiva de la disciplina y la situación en el contexto actual, lo que permite retomar desde los orígenes de la misma, los avances, los éxitos y los desaciertos para comprender el entorno actual así como los desafíos futuros.

La reflexión para pensar el componente internacional en el marco socioprofesional puede guiarse con las preguntas siguientes:

1. ¿Cómo se interpreta la globalización de la economía en la profesión?

2. ¿De qué manera la globalización ha transformado e impactado la educación superior, la disciplina y la profesión?

3. ¿Cuáles son los nexos de la carrera con otras culturas y con otros contextos? ¿Cómo se han construido esos enlaces? ¿De qué manera se desea fortalecerlos? ¿Cuáles nuevos vínculos académicos y culturales se desea explorar? ¿Por qué?

4. ¿Cuáles son las proyecciones para el intercambio cultural de esta carrera en el ámbito internacional?

5. ¿Cuáles beneficios académicos, sociales-culturales, políticos y económicos espera obtener la carrera con la internacionalización de su currículum?

6. ¿Cómo se benefician, con la internacionalización del currículum, las diferentes poblaciones vinculadas con la carrera (docentes, estudiantes, personal técnico y administrativo, personas egresadas, sector empleador)?

7. ¿Cuáles aportes dará esta carrera a las otras homólogas y a las universidades con las que se establecerán contactos internacionales? 
8. ¿Cuáles habilidades, destrezas y conocimientos necesitan los/as profesionales para ejercer y desarrollarse en otros contextos culturales? ¿Cuáles prácticas profesionales son necesarias para salir del país?

9. ¿Cuáles son las normas internacionalmente aceptadas para el ejercicio de su profesión?

10. ¿Cuáles aportes concretos puede hacer una persona profesional en esta disciplina en el ámbito profesional-internacional?

11. ¿Cuáles nuevas áreas de inserción internacional pueden tener las personas de esta profesión?

\subsubsection{Marco epistemológico}

Bolaños et al., (1999) sugieren que en este marco se reflexione sobre: "la validez y la legitimidad de la disciplina, desde su fundamentación" (p. 190). Esto quiere decir que es necesario comprender cómo se gesta el conocimiento de esta disciplina.

Algunas de las reflexiones que se proponen son las siguientes:

1. ¿Cuáles fenómenos de carácter internacional propone investigar y transformar?

2. ¿Es el conocimiento de esta disciplina un conocimiento universal? ¿Cómo se construye el conocimiento en esta disciplina, en contextos interculturales? ¿De qué manera el conocimiento de esta disciplina se puede fortalecer en otros escenarios ?

3. ¿Es posible investigar, en esta disciplina, mediante estudios comparativos, interdisciplinarios, internacionales e interculturales?

4. ¿Cuáles son las características de la comunidad internacional que investiga en esta disciplina?

5. ¿De qué manera se favorece con la internacionalización del currículum, la interdisciplinariedad, multidisciplinariedad y la transdiciplinariedad de las ofertas educativas?

\subsubsection{Propósitos de la carrera}

Siguiendo a las autoras citadas (1999), en este apartado se busca que la carrera explicite lo que se pretende con la formación; para ello, se sugieren las preguntas siguientes:

1. ¿De qué maneras se beneficiará la universidad, el país y la región, con una formación con carácter internacional de los y las profesionales?

2. ¿Qué se pretende con la formación internacional? 
3. ¿Cuáles criterios de internacionalización se consideran viables para la formación en esta disciplina?

\subsubsection{Selección de saberes}

Bolaños et al., (1999), indican que en este momento de la reflexión curricular se sistematizan: "las herramientas cognitivas que requiere el profesional y la profesional, así como los aspectos relacionados con las destrezas, actitudes y valores que se desea fomentar" (p. 192). Este apartado es uno de los más claros para el cuerpo docente, pues es donde se seleccionan los saberes que dan sustento a su disciplina y que también provocan una tensión entre distintos intereses profesionales y disciplinares.

Las preguntas sugeridas son las siguientes:

1. ¿Cuáles herramientas cognitivas y cuáles valores requieren las personas profesionales para intervenir, investigar y transformar el objeto de estudio de la disciplina profesional en el ámbito internacional?

2. ¿Se incorporan cursos que fortalezcan el dominio de otro (s) idioma(s)?

3. ¿Qué cantidad de cursos o experiencias de aprendizaje serán necesarios en este plan de estudios?

4. ¿En cuáles niveles del plan de estudio se incorporan los criterios de internacionalización que ha determinado la carrera?

5. ¿Se incorporan estudios de otras culturas en el plan de estudios? ¿De qué manera?

\subsubsection{Enfoque pedagógico}

Es el momento del diseño curricular cuando se reflexiona acerca de la concepción que se tiene del aprendizaje y la enseñanza (Bolaños, et. al., p. 192). Recordando nuevamente el principio de flexibilidad y de apertura al cambio, el enfoque pedagógico debe permitir la experimentación, la innovación, la flexibilidad y la participación de distintos actores en el proceso de formación. El intercambio de experiencias de aprendizaje y de expresiones de la cultura debe ser parte de la formación internacional.

Se proponen las preguntas siguientes:

1. ¿Cuál enfoque pedagógico permite mayor flexibilidad en el currículo?

2. ¿Cuáles estrategias didácticas favorecen el intercambio de experiencias educativas y culturales? 
3. ¿Cómo se incorporan las Tecnologías de la Información y de la Comunicación en este proceso de aprendizaje?

4. ¿Cómo se va a evaluar el aprovechamiento de las experiencias de internacionalización?

\subsubsection{Organización}

En este aspecto se deben estructurar y ordenar los saberes para facilitar la formación profesional, tal y como se ha venido reflexionando.

Se proponen las preguntas siguientes orientadoras:

1. ¿Cuáles son los criterios para convalidar los aprendizajes de docentes y estudiantes obtenidos en sus intercambios culturales y académicos?

2. ¿Cuáles estrategias de intercambio de información son necesarias para agilizar la gestión de la propuesta curricular con las instituciones internacionales y las demás instancias y personas involucradas?

3. ¿Cuáles criterios deben considerarse para equiparar los títulos obtenidos en el extranjero?

4. ¿Qué porcentaje del plan de estudios puede ser equiparado y convalidado?

5. ¿Cuáles áreas del plan de estudios pueden ser aprendidas en otros contextos culturales y sociales?

6. ¿Cuáles requisitos deben cumplir el estudiantado y el cuerpo de docentes que realicen procesos de aprendizaje en el extranjero?

7. ¿Cómo se articulan los proyectos de investigación con los criterios de internacionalización definidos por la carrera?

8. ¿Cómo se articulan los proyectos de acción social y/o extensión docente con los criterios de internacionalización definidos por la carrera?

\subsubsection{Administración, ejecución y seguimiento}

En este momento del diseño curricular: "se clarifican los aspectos estructurales y prácticos de la propuesta, para garantizar el cumplimiento de los propósitos establecidos". También, se establecen los mecanismos de seguimiento y de ajuste que se crean convenientes (Bolaños et. al, p. 193). Por su parte, la gestión del currículo se refiere a la posibilidad de implementar y monitorear el desarrollo del currículo, e implica lograr la 
articulación de los diversos elementos de la internacionalización en este plan educativo, para lograr el fin deseado.

Por lo tanto, puede interpretarse como la posibilidad de implementar y promover las estrategias que propicien la temática de la internacionalización, de acuerdo con los criterios establecidos por la unidad académica o institución.

Se proponen las siguientes interrogantes:

- ¿Es posible la traducción, a otros idiomas de interés para la carrera, de toda la información referente al plan de estudios?

- ¿Es factible la movilidad estudiantil y del personal docente?

- ¿De qué maneras se pueden aprovechar los recursos que ofrecen las instancias universitarias para favorecer el proceso de internacionalización del currículum?

- ¿Cuáles recursos son necesarios para lograr la movilidad estudiantil y de docentes?

- ¿Cuáles procesos de capacitación internacional se pueden realizar para el profesorado y el estudiantado de la institución?

- ¿De qué manera se hará el seguimiento y la evaluación de la propuesta curricular?

- ¿Cuáles mecanismos se van a implementar para realizar los ajustes necesarios a la propuesta de internacionalización?

Todo lo anterior expresa los criterios que desde la perspectiva de un currículum pertinente, coherente y consistente debe tener una propuesta que intente ser internacional.

\section{Conclusiones}

Mediante la internacionalización del currículum, las instituciones de educación superior pueden lograr un balance entre los intereses nacionales, tanto de la universidad como del país, y los intereses o motivos de proyección cultural y académica que priorice la institución.

Las carreras con una oferta educativa internacionalizada pueden fortalecer su disciplina y proyectarse en otros ámbitos culturales, políticos, económicos a nivel internacional, lo que redunda en beneficios para todas las poblaciones vinculadas con el proceso de enseñanza y aprendizaje.

La incorporación de los criterios de internacionalización al currículum de las carreras implica un riguroso proceso de planeación, tanto a nivel de la administración y gestión de la 
carrera propiamente, como a nivel de la organización general de la universidad, lo que posibilita un mejor aprovechamiento de los recursos y mayor articulación de los procesos,

La gestión curricular de un plan de estudios con criterios de internacionalización debe, en primera instancia, ser flexible para incorporar las diferentes posibilidades de desarrollo individual y colectivo de estudiantes y docentes y, además, se recomienda que tome en cuenta los cambios en las formas de organización y de administración de los recursos.

La reflexión, la discusión y el análisis sobre las implicaciones que tiene incorporar estos criterios en un plan de estudios es un paso indispensable para lograr la coherencia entre los fines de la institución y el desarrollo de la propuesta educativa que, como se ha mantenido a lo largo de este artículo, debe ser un componente articulado en todo el proceso formativo.

\section{Referencias}

Alfaro L., Rosalinda (1996). Pensamiento crítico, un enfoque práctico. Barcelona: Editorial Masson S.A.

Beneitone, Pablo, Esquetini, César, González Julia, Maletá, Maida, Siufi, Gabriela y Wagenaar, Robert. (2007). Reflexiones y Perspectivas de la educación Superior (Informe Final Proyecto Tunnig América latina 2004-2007). Bilbao: Universidad de Deusto Universidad de Groningen

Beneitone, Pablo. (2008). Internacionalización del Currículo en América Latina y el Caribe. (Argentina). Presentación en power point en el Foro sobre Internacionalización de la Educación Superior, realizado en la Universidad del Cauca, en marzo de 2008. Recuperado el 11 de junio, 2010 de: http://www.ascun.org.co/eventos/forointernacionalizacion/pablobeneitone.pdf

Bolaños, Carolina, Vargas, Alicia y Velásquez, Leda. (1999). Hacia un diseño curricular alternativo en la educación superior. Revista Educación, 23(Especial), 185,194. $\begin{array}{llllll}\text { Recuperado el } & 24 & \text { de } & \text { abril } & \text { de } & 2010\end{array}$ http://www.cea.ucr.ac.cr/diea/documentos/Dise\%f10\%20curricular\%20alternativo.pdf

Canudas, Luis F. (2006). El currículum de estudios en la enseñanza superior. $\begin{array}{lllll}\text { Recuperado el } & 3 & \text { junio, } & 2010 & \text { de }\end{array}$ http://www.anuies.mx/servicios/p anuies/publicaciones/revsup/res002/txt2.htm\#2

Camillioni Alicia. (2010). Gestión curricular en las instituciones de Educación Superior. Conferencia de Red Interinstitucional de Formación Docente, RIFED. 2-9-2010. Universidad de Costa Rica

Didou, Sylvie. (2007) La internacionalización de la educación superior en América Latina; oportunidades y desafíos. Conferencia del 21 de agosto del 2007 Universidad Nacional de Córdoba, Argentina. 
Facione, Peter. (2007. Pensamiento crítico: ¿Qué es y por qué es importante? Insight Assessment. California Academic Press. Recuperado el 3 de Julio de 2010 de http://www.eduteka.org/PensamientoCriticoFacione.php

Fedorov, Andrei. (2009). Beneficios y riesgos de la internacionalización de la educación superior. Informa TEC, (289). Recuperado 02 de julio, 2010 de http://www.tec.cr/prensa/Informatec/2009/abril/n20.html

Gacel-Avila, Jocelyne. (s.f.). La internacionalización de las universidades mexicanas. Políticas y estrategias institucionales. México: ANUIES. Recuperado 04 de junio, 2010 http://www.ses.unam.mx/curso2009/materiales/m6/LecturaComplementaria/M6 Comp lemetaria Gacel.pdf

Harris, Suzy. (2008). Internationalising the University. Educational Philosophy and Theory, 40(2), 346-357.

International Association of Universities. (2005). Key results: 2005 IAU Global Survey on Internationalization of Higher Education. Recuperado 02 julio, 2010 de http://www.iau-aiu.net/internationalization/pdf/Key results 2005.pdf

Japan Society for the Promotion of Science, JSPS. (s.f.). Innovative Models for Promoting the Internationalization of Japanese Universities (Interim Report). Recuperado 02 de julio, 2010 de http://www.jsps.go.jp/english/e-bilat/e-ukokusen/program org/interimreport/4-2.pdf

Knight, Jane. (s.f.) Internacionalización y aseguramiento de la calidad: conceptos. Calidad e internacionalización en la educación superior. México: ANUIES.

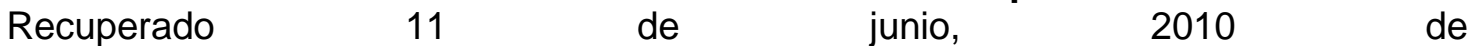
http://www.anuies.mx/servicios/d estrategicos/libros/lib62/indice.html

Knight, Jane. (2006). Global University Network for Innovation, GUNI. Recuperado el 25 de junio de 2010 de http://www.iauaiu.net/internationalization/pdf/Key results 2005.pdf

Leask, Betty. (1999). Internationalisation of the currículum: Key challenges and strategies. Flexible Learning Centre, University of South Australia. Recuperado 02 de setiembre, $2010 \quad$ de http://www.unisa.edu.au/ltu/staff/practice/internationalisation/documents/currículumleask.pdf

Madera, Inmaculada. (2005) Un nuevo paradigma educativo: la internacionalización del currículum en la era global. Universidad APEC República Dominicana Ponencia en el Cuarto encuentro de Educación y Pensamiento, 6 al 8 de octubre del 2005. Republica Dominicana. Recuperado 5 de junio, 2010 de http://ofdp rd.tripod.com/encuentro4/apec.html 
Orozco F., Bertha. (2009). Currículum: experiencias y configuraciones conceptuales en México. México: Instituto de investigaciones sobre la Universidad IISUE. Plaza y Valdés Editores

Pedregal, Rafael. (2003). La internacionalización de la educación superior en América del Norte ante los retos del TLCAN: un estudio comparativo. Tesis profesional para obtener el título de Maestría en Estudios sobre Estados Unidos de Norteamérica. Universidad de las Américas. Puebla, México. Recuperado 18 junio, 2010 de http://catarina.udlap.mx/u dl a/tales/documentos/mes/pedregal c r/

Perdomo, Rocío. (s.f.). Propuesta para un modelo curricular flexible. Escuela de Ciencias de la Educación de la Universidad Autónoma de Baja California. Recuperado 44 de junio, $\quad 2010$ de http://www.anuies.mx/servicios/p anuies/publicaciones/revsup/res085/txt10.htm\#3

Qiang, Zha. (2003). Internationalization of Higher Education: towards a conceptual framework. Policity Futures in Education, 1(2). Recuperado el 04 de junio, 2010 de http://www.unal.edu.co/ori/red-orion/docs/Conceptual-FrameworkInternationalization.pdf

Ruiz, José María. (2005). Teoría del currículum: Teoría, desarrollo e innovación curricular. Madrid: Editorial Universitaria.

Tünnermann, Carlos. (s.f.) La universidad en el contexto de la internacionalización y la multiculturalidad. $\quad$ Recuperado 02 julio, 2010 de http://www.congresoretosyexpectativas.udg.mx/Congreso\%206/Conferencias\%20Mag istrales/Mesa4/carlostunnerman.pdf

Universidad de Costa Rica. Consejo Universitario. (2008). Políticas de la Universidad de Costa Rica para los años 2010-2014. Recuperado el 9 de abril de 2010 de http://cu.ucr.ac.cr/normativ/politicas institucionales 2010-2014

Universidad de Costa Rica. Oficina de Asuntos Internacionales. (s.f.). Sitio de la Oficina de Asuntos Internacionales y Cooperación Externa. Recuperado el 9 de setiembre de 2010 de http://www.oaice.ucr.ac.cr/

Upegui, María Eugenia. (2007). La flexibilidad curricular y el contexto socioeconómico. Antioquia, Colombia: Grupo Interdisciplinario de Investigación en Currículo. Universidad de Antioquia

Zarur, Xiomara. (2010). La internacionalización del currículo: una herramienta fundamental para la transformación institucional. Seminario "Internacionalización de la educación superior con una visión para las instituciones técnicas profesionales y tecnológicas". Bogotá, 6 de mayo de 2010. Recuperado 02 de setiembre, 2010 de http://www.colombiaaprende.edu.co/html/estudiantesuperior/1608/articles231231 archivo pdf1.pdf 